\title{
Glycolysis inhibitor 2-deoxy-D-glucose suppresses carcinogen-induced rat hepatocarcinogenesis by restricting cancer cell metabolism
}

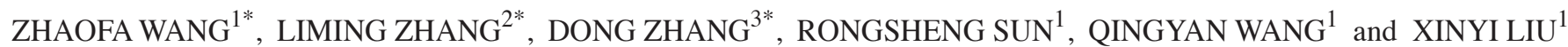 \\ ${ }^{1}$ Department of General Surgery, Weihai Second Municipal Hospital, Weihai Women and Children's Hospital, \\ Weihai Hospital Affiliated to Qingdao University, Weihai, Shandong 264200; \\ ${ }^{2}$ Department of Otorhinolaryngology, Weihaiwei People's Hospital, Weihai, Shandong 264000; \\ ${ }^{3}$ Department of General Surgery, Huadong Hospital Affiliated to Fudan University, Shanghai 200040, P.R. China
}

Received June 28, 2014; Accepted October 24, 2014

DOI: $10.3892 / \mathrm{mmr} .2014 .2945$

\begin{abstract}
The abnormal metabolism of cancer cells is a crucial feature of tumors and provides promising therapeutic targets for cancer treatments. Aerobic glycolysis in cancer cells, termed the Warburg effect, is a highlighted characteristic of cancer-specific metabolism. However, the effect of glycolysis inhibition on hepatocarcinogenesis remains to be elucidated. In the present study, the effects of the glycolysis inhibitor 2-deoxy-D-glucose (2-DG) on the $\mathrm{N}$-diethylnitrosamine (DEN)-induced rat hepatocarcinoma model and its underlying mechanisms were investigated. It was observed that 2-DG significantly delayed hepatocarcinogenesis and effectively prolonged survival time in the DEN-treated rats. The glycolysis inhibitor, 2-DG prominently decreased cell proliferation and increased cell apoptosis in the DEN-induced rat hepatoma and had no evident impact on the pericarcinomatous liver tissues. Further investigation revealed that 2-DG resulted in a reduction of glycolysis products, the compensatory increase of hexokinase 2 expression and a decrease in 6-phosphofructo-2-kinase, pyruvate kinase M2 and lactate dehydrogenase A expression in the hepatoma tissues. The inhibition of glycolysis further suppressed the tricarboxylic acid cycle, fatty acid and cholesterol biosynthesis and ATP production, while it promoted autophagic activation. In addition, the in vitro study demonstrated that hypoxia,
\end{abstract}

Correspondence to: Professor Xinyi Liu, Department of General Surgery, Weihai Second Municipal Hospital, Weihai Women and Children's Hospital, Weihai Hospital Affiliated to Qingdao University, Weihai, 51 Guangming Road, Shandong 264200, P.R. China

E-mail: liuxinyi_weihai@126.com

*Contributed equally

Key words: hepatocarcinogenesis, glycolysis, 2-deoxy-D-glucose, cancer cell metabolism an important factor in the tumor microenvironment, may assist in increasing 2-DG-induced inhibition of cell viability, cell cycle retardation and the decrease of colony formation ability in hepatoma cells. Taken together, the present results suggested that 2-DG may inhibit hepatocarcinogenesis in the DEN-treated rats via restricting cancer cell metabolism. This finding provides a promising measure in the prevention and treatment of hepatoma.

\section{Introduction}

Hepatocellular carcinoma (HCC) is one of the most common types of malignancy worldwide. In males, it is the fifth most common cancer and the second leading cause of cancer-associated mortality (1). The low rate of early diagnosis and rapid tumor development lead to a poor prognosis and high mortality in HCC patients (2). Therefore, investigating the mechanism of hepatocarcinogenesis and further examining effective therapeutic targets are important for HCC prevention and treatment.

The abnormal metabolism of cancer cells has been broadly investigated and is considered a potential target to treat cancer $(3,4)$. The Warburg effect, which is the switch from oxidative phosphorylation to aerobic glycolysis, is frequently observed in various types of cancer cells and is known as a hallmark of cancer development $(5,6)$. Enhanced aerobic glycolysis affords a steady supply of metabolic production essential for biosynthesis and energy generation and may also affect various related metabolic pathways that are associated with numerous biological processes, including cell proliferation, apoptosis and invasion (7). HCC treatments, which target aerobic glycolysis have been receiving increasing attention.

2-deoxy-D-glucose (2-DG), a glucose analogue able to suppress glycolysis by competitively inhibiting hexokinase 2 (HK2), has an impact on HCC cell lines (8) and is reported to inhibit the growth of transplanted HCCs $(9,10)$. However, whether it has effects on the development of primary liver tumors remains to be elucidated. In the present study, the effects of 2-DG on the N-diethylnitrosamine (DEN)-induced rat hepatocarcinoma model and its mechanisms were investigated. 


\section{Materials and methods}

DEN and 2-DG model in the rat. Male 10-12 week old Sprague Dawley rats, weighing 220-240 g were purchased from Shanghai Slaccas Laboratory Animal Company Limited (Shanghai, China) and were maintained at an animal facility under pathogen-free conditions. All rats received humane care according to the guidelines of the Chinese Association for Laboratory Animal Sciences (Beijing, China). The present study was approved by the ethics committee of Medical College of Qingdao University, (Qingdao, China).

To induce HCC, DEN at a dilution of 1/10,000 (95 mg/l; Sigma-Aldrich, St. Louis, MO, USA), was added to the drinking water of rats for 13 weeks. Subsequently, the water was replaced by normal sterile water and certain rats received $0.75 \mathrm{~g} / \mathrm{kg}$ 2-DG (Sigma-Aldrich), dissolved in normal saline solution via intraperitoneal (IP) injection once every 3 days until the end of the 17th week after initial DEN administration. A total of 30 rats (control group, $n=5$; 2 -DG group, $n=5$; DEN group, $\mathrm{n}=10$; $\mathrm{DEN}+2-\mathrm{DG}$ group, $\mathrm{n}=10$ ) were kept and assessed for survival time and 20 rats (DEN group, $n=10$; $D E N+2-D G$ group, $\mathrm{n}=10$ ) were sacrificed through cervical dislocation at 17 weeks in order to observe their primary liver tumors. The tumors were measured with a micrometer up to a maximum diameter of $3 \mathrm{~mm}$. The tumor volumes were calculated using the following formula: Volume $=a b^{2} / 2(' a$ ' is the maximal diameter and ' $b$ ' was the minimal diameter).

Liver sections were soaked in $10 \%$ neutral-buffered formalin for hematoxylin and eosin (H\&E) and immunohistochemical (IHC) staining, preserved in RNA Later (Qiagen GmbH, Hilden, Germany) for detecting mRNA expression and snap-frozen in liquid nitrogen for detecting protein expression.

$H \& E$ and IHC staining. The paraffin-embedded liver sections were subjected to H\&E staining for histopathological analysis. The primary IHC antibodies included: Ki67 (polyclonal rabbit anti-rat; 1:1,000; ab16667; Abcam, Cambridge, UK), Actived-Caspase-3 (polyclonal rabbit anti-rat; 1:100; BS7004; Bioworld Technology, St.Louis Park, MN, USA) Beclin-1 (polyclonal rabbit anti-rat; 1:100; 3738; Cell Signaling Technology, Inc., Beverly, MA, USA) and microtubule-associated protein 1A/1B-light chain 3 (LC3; polyclonal rabbit anti-rat; 1:200; 4108; Cell Signalling Technology, Inc.).

Western blotting. The lysates of liver tumors were subjected to SDS-PAGE. The protein blots were incubated with specific primary antibodies, including anti-proliferating cell nuclear antigen (PCNA; polyclonal rabbit anti-rat; 1:1,000; Bs6438; Bioworld Technology), anti-cyclin D1 (polyclonal rabbit anti-rat; 1:1,000; BS6532; Bioworld Technology), anti-activated-caspase 3 (polyclonal rabbit anti-rat; 1:1,000; BS7004; Bioworld Technology), anti-LC3 (polyclonal rabbit anti-rat; 1:1,000; catalogue number, 4108; Cell Signalling Technology) and anti-P62 (polyclonal rabbit anti-rat; 1:1,000; 5114; Cell Signaling Technology, Inc.) and subsequently incubated with polyclonal goat anti-rabbit IgG horseradish peroxidase-conjugated (heavy and light chain) secondary antibody $(1: 20,000$; BS10350; Bioworld Technology) and chemiluminescent substrates. Anti- $\beta$-actin (polyclonal rabbit anti-rat; 1:10,000;
AP0060; Bioworld Technology) was used to confirm equal protein loading.

Biochemical analysis. The levels of glucose-6-phosphate (G-6-P), acetyl-CoA, citrate, isocitrate (Biovision, Mountain View, CA, USA), pyruvate, lactic acid, ATP (Nanjing Jiancheng Bioengineering Institute, Nanjing, China), 3-hydroxy-3-methylglutaryl-coenzyme A (HMG-CoA; Shanghai Enzyme-linked Biotechnology, Co., Ltd, Shanghai, China) and malonyl-CoA (Shanghai Yu Ping Biotechnology Limited Company, Shanghai, China) were measured using assay kits, according to the manufacturer's instructions.

Reverse transcription-quantitative polymerase chain reaction (RT-qPCR). Total RNAs of HCC tissues in the rats were extracted using TRIzol reagent (Invitrogen Life Technologies, Carlsbad, CA, USA) and were further treated with RNase-free DNase (Promega Corporation, Madison, WI, USA). The cDNA was synthesized using the RevertAid First-Strand cDNA Synthesis kit (Fermentas, Vilnius, Lithuania). RT-qPCR was performed using a Roche LightCycler 480 system (Roche Applied Science, Indianapolis, IN, USA). The specific primers used to analyze gene expression were as follows: HK2, forward 5'-TTTGGTCTCGTGGACTAAGGG-3' and reverse 5'-ACCACGGCCACAATGTCAAT-3'; 6-phosphofructo-2-kinase (6PF2K), forward 5'-AAAGGCATTGCCGCCCGGAAGTG-3' and reverse 5'-TGTAATACGACTCACTATA-3'; pyruvate kinase M2 (PKM2), forward 5'-GGTCATCTGTGCCAACCAGA-3' and reverse 5'-AGGGACAGGGGCTAGAA GAG-3' and lactate dehydrogenase A (LDHA), forward 5'-GGTCATCTGTGCCAACCAGA-3' and reverse 5'-AGGGACAGGGGCTAGAAGAG-3'. Fold change in gene expression was determined by normalizing to endogenous $\beta$-actin, with the following primer sequence: Forward 5'-CTCTATCCTGGCCTCACT GTCCACC-3' and reverse 5'-AAACGCAGCTCAGTAACAGTCCGC-3'.

Cell culture. Human HCC cell lines SMMC7721 and HepG2 were cultured in Dulbecco's modified Eagle's medium containing $10 \%$ fetal bovine serum, $100 \mathrm{U} / \mathrm{ml}$ penicillin and $100 \mu \mathrm{g} / \mathrm{ml}$ streptomycin, which were all purchased from Gibco-BRL (Carlsbad, CA, USA), at $37^{\circ} \mathrm{C}$ under humidified air containing $5 \% \mathrm{CO}_{2}$. For the hypoxic condition, cells were cultured in a tri-gas incubator (Sanyo, Osaka, Japan) maintained at $3 \% \mathrm{O}_{2}, 5 \% \mathrm{CO}_{2}$ and $92 \% \mathrm{~N}_{2}$. 2-DG was dissolved in tri-distilled water as stock solution (1 $\mathrm{M})$ and was then added into the media to the final concentration $(10 \mathrm{mM})$.

Cell viability detection. Cell viability was evaluated using the Cell Counting kit-8 (CCK-8; Dojingo Laboratories, Kumamoto, Japan). The cells in exponential growth period were plated in 96-well culture plates $(1,000$ cells/well) for different culture periods. Subsequently, CCK-8 solution was added to each well. Incubation was performed at $37^{\circ} \mathrm{C}$ for $1 \mathrm{~h}$. The optical density of each well was measured at $450 \mathrm{~nm}$ using an ELISA reader (ELx808; Bio-Tek Instruments, Winooski, VT, USA).

Cell cycle detection. SMMC7721 and HepG2 cells were plated in 6 -well culture plates $\left(5 \times 10^{5}\right.$ cells/well) and treated 
A

\section{Experimental Protocol:}

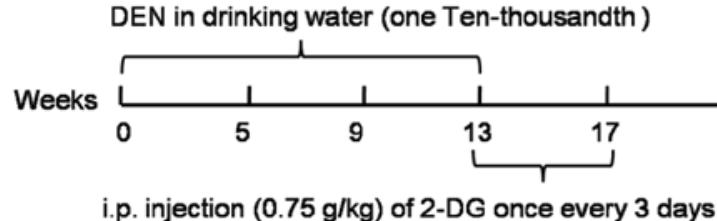

B

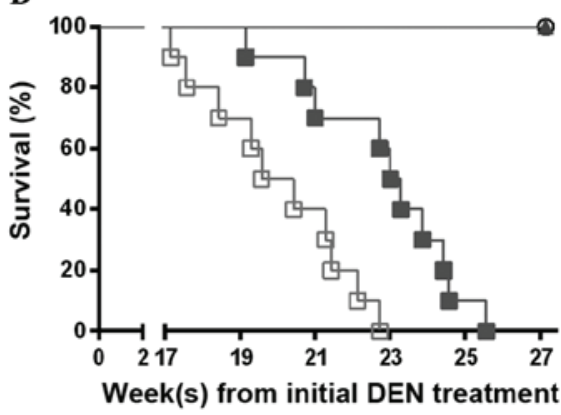

$\mathbf{E}$

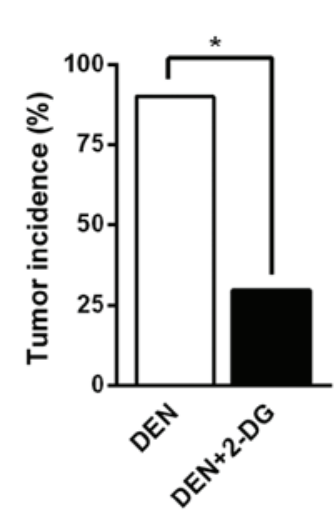

D

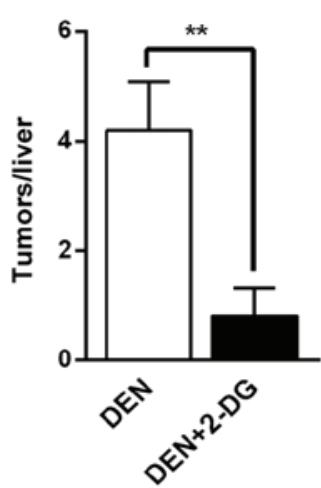

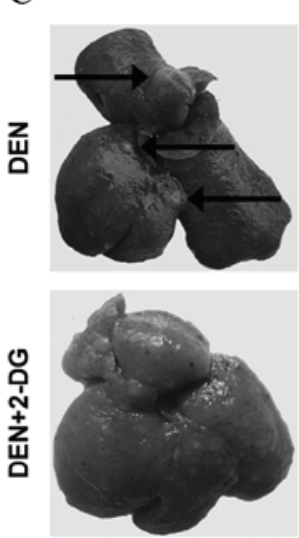

H\&E

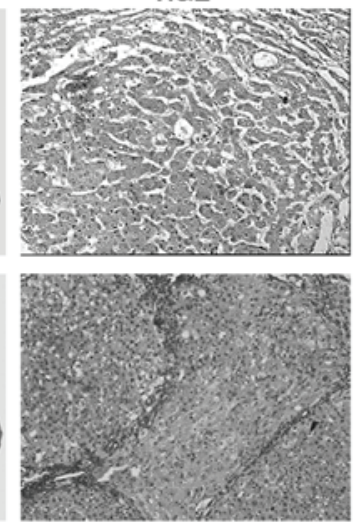

$\vartheta$ Control $(n=5)$

$\pm 2-D G(n=5)$

も $\operatorname{DEN}(\mathrm{n}=10)$

- $D E N+2-D G(n=10)]$ *

Figure 1. 2-DG suppresses hepatocarcinogenesis in DEN-treated rats. (A) Diagram of the experimental protocol. DEN water $(1 / 10,000 ; 95 \mathrm{mg} / \mathrm{l})$ was administered to rats for 13 weeks and then replaced by normal water. Rats in the DEN+2-DG group received extra 2-DG solution ( $0.75 \mathrm{~g} / \mathrm{kg}$ body weight) by intraperitoneal injection once every 3 days (14-17th week). (B) Survival curves of the DEN and DEN+2-DG groups ("** $\mathrm{P}<0.01$ vs. DEN group). (C) Rat livers of DEN and DEN+2-DG groups at 17 weeks. Arrows indicate tumor. Liver sections were stained with hematoxylin \& eosin (magnification, x100). (D) Tumor incidence, (E) multiplicity and (F) max tumor volume of DEN and DEN+2-DG groups at 17 weeks. Data are presented as the mean \pm standard error of the mean $\left({ }^{*} \mathrm{P}<0.05\right.$ and ${ }^{* *} \mathrm{P}<0.01$ vs. DEN group). DEN, N-diethylnitrosamine; 2-DG, 2-deoxy-D-glucose.

as described above. After $24 \mathrm{~h}$ treatment, the cells were centrifuged at $500 \mathrm{x} \mathrm{g}$ for $5 \mathrm{~min}$. The cells were washed with pre-cooled phosphate-buffered saline $(\mathrm{pH} 7.4)$ twice and then fixed in $70 \%$ alcohol and stained with propidium iodide (Sigma-Aldrich). Analysis of cell cycle distribution was performed using a flow cytometer (FACSCalibur; BD Biosciences, Franklin Lakes, NJ, USA) in accordance with the manufacturer's instructions.

Colony formation assay. Exponentially growing cells were plated in 6-well culture plates (200 cells/well) and treated using the indicated methods. The plates were maintained at $37^{\circ} \mathrm{C}$ in $20 \% \mathrm{O}_{2}$ or $3 \% \mathrm{O}_{2}$ for 2 weeks. Following fixation in paraformaldehyde, the colonies were stained with crystal violet for $10 \mathrm{~min}$. Subsequently, images were captured with a digital camera (Sony H7; Sony Corporation, Tokyo, Japan) and the visible colonies were recorded.

Statistical analysis. Data are presented as the mean \pm standard error of the mean. Statistical significance was determined by Student's t-test and one-way analysis of variance. Tumor incidence was analyzed by Fisher's exact test. Survival curves were analyzed by log-rank test. $\mathrm{P}<0.05$ was considered to indicate a statistically significant difference. Statistical analyses were performed using GraphPad Prism 6.04 software (GraphPad Software Inc., La Jolla, CA, USA).

\section{Results}

2-DG inhibits DEN-induced hepatocarcinogenesis in the rat. To examine the effects of 2-DG on primary liver tumor development, a classical DEN rat model was used, which resembles human hepatocarcinoma in rats. In this model, the majority of the rats $(>90 \%)$ developed liver tumor nodules by the end of the 17 th week after initial DEN administration $(11,12)$. The rats in the $\mathrm{DEN}+2-\mathrm{DG}$ group were administered 2-DG by IP injection from the 14th-17th week after the initial DEN administration (Fig. 1A).

In the cohort of DEN-treated rats monitored for survival time, the DEN+2-DG group exhibited a longer mean survival time compared with that of the DEN group (Fig. 1B). Further investigation revealed that, at 17 weeks, $90 \%$ of the rats in the DEN group had typical HCC nodules, however, the HCC incidence in the DEN+2-DG group was only 30\% (Fig. 1C and D). In addition, the DEN group exhibited a 5-fold higher HCC multiplicity $(4.2 \pm 0.9$ versus $0.8 \pm 0.5)$ and an 18.7 -fold higher maximum tumor volume $\left(321.5 \pm 127.4\right.$ versus $\left.17.2 \pm 13.1 \mathrm{~mm}^{3}\right)$ as compared with those of the DEN+2-DG group (Fig. 1E and F). The present results demonstrated that 2-DG significantly suppressed hepatocarcinogenesis in the DEN-treated rats.

2-DG suppresses cell proliferation and promotes cell apoptosis in DEN-induced HCC. Subsequently, the present study 
A
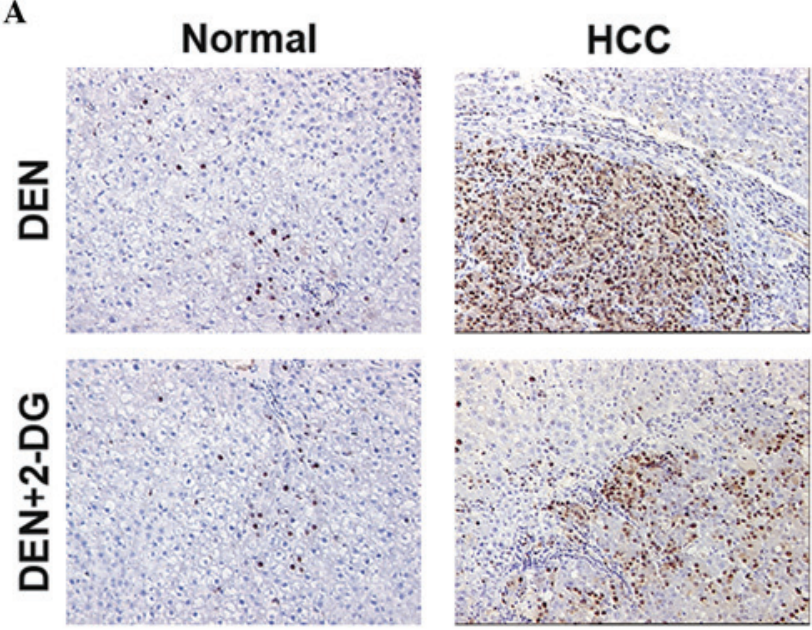

B
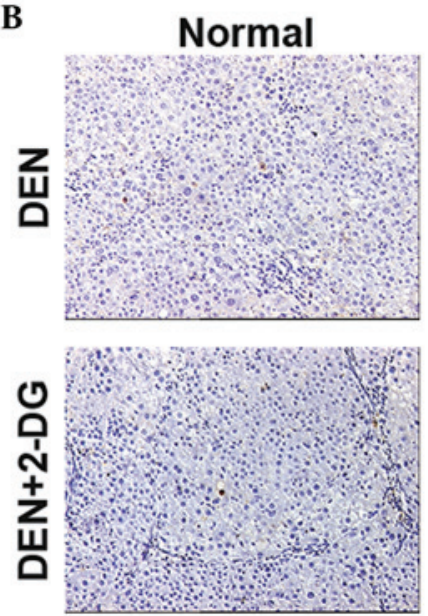

HCC
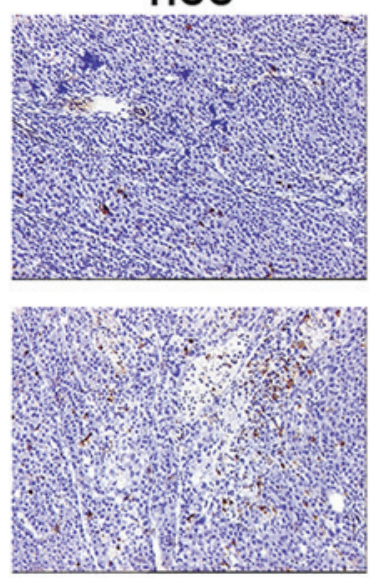

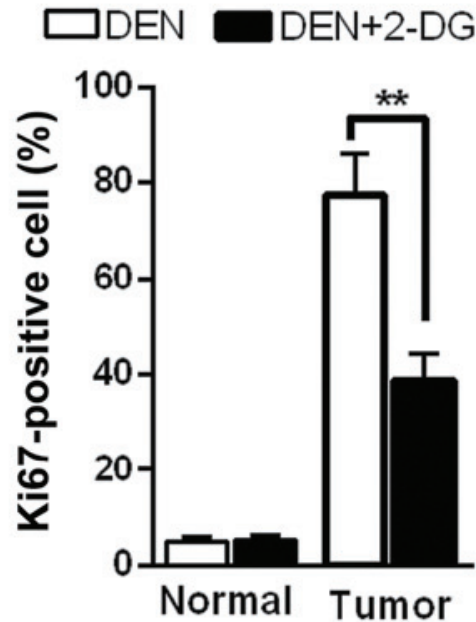

$\square D E N \square D E N+2-D G$

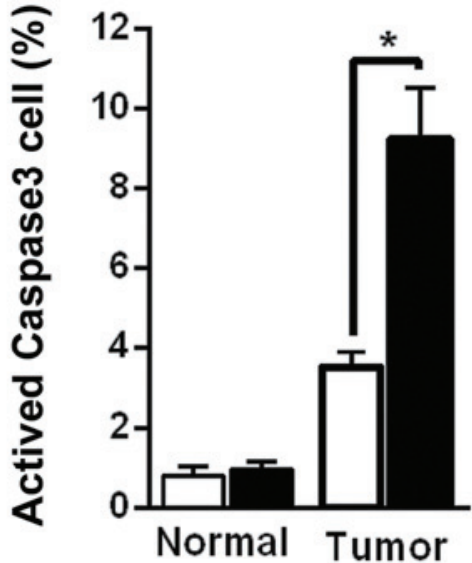

C

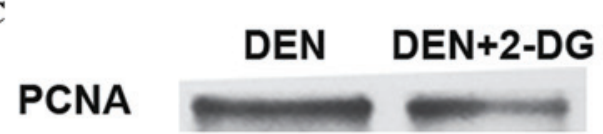

Cyclin D1

Actin

\section{D actived
Caspase3 \\ Cleaved PARP1} DEN DEN+2-DG

Actin

Figure 2. 2-DG suppresses tumor cell proliferation and promotes tumor cell apoptosis in DEN-treated rat livers. (A and B) Immunohistochemistry staining for (A) Ki67 and (B) activated caspase 3 in the HCC and pericarcinous tissues of DEN and DEN+2-DG groups at 17 weeks (magnification, x100). Graphs represent the percentages of positive cells (right panel). Data are presented as the mean \pm standard error of the mean ( $\mathrm{P}<0.05$ and ${ }^{* *} \mathrm{P}<0.01$ vs. $\mathrm{DEN}$ group). (C and D) Cell lysates obtained from HCC tissues of DEN and DEN+2-DG groups at 17 weeks were immunoblotted with the indicated antibodies ( $\mathrm{n}=3$ ). DEN, N-diethylnitrosamine; 2-DG, 2-deoxy-D-glucose; PCNA. proliferating cell nuclear antigen; HCC, hepatocellular carcinoma; PARP1, poly ADP-ribose polymerase 1 .

aimed to examine how 2-DG inhibits DEN-induced hepatocarcinogenesis in the rat. Cell proliferation and apoptosis were detected in the DEN-treated rat livers at 17 weeks. IHC staining revealed that 2-DG markedly reduced cell proliferation (Ki67-positive cells) in the HCC tissues, but had no significant effect on the normal liver cells in the pericarcinous tissues (Fig. 2A). Additionally, treatment with 2-DG resulted in increased cell apoptosis (activated caspase 3-positive cells) in the HCC tissues, but not in the pericarcinous tissues (Fig. 2B). Immunoblot analyses also revealed similar results. The data demonstrated that HCC tissues of the DEN+2-DG group had lower levels of PCNA and cyclin D1, indicators of 
DEN $\square$ DEN+2-DG

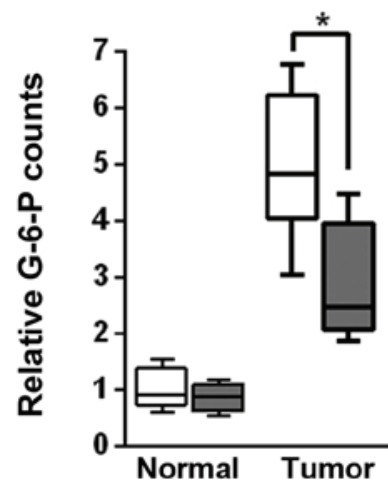

B

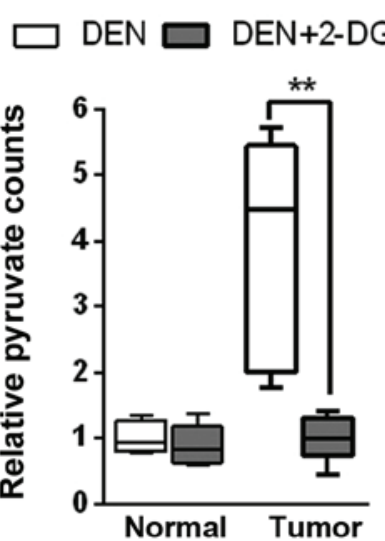

C

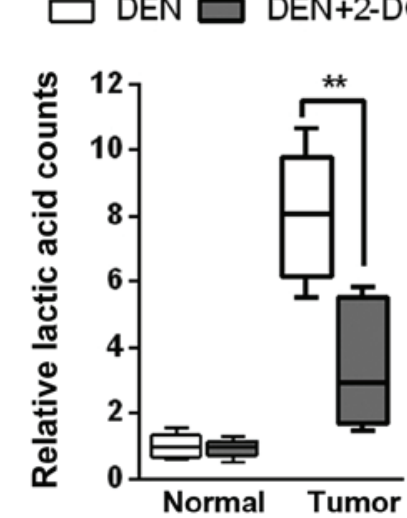

G

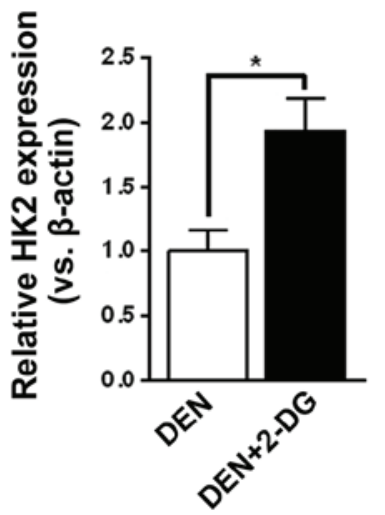

E

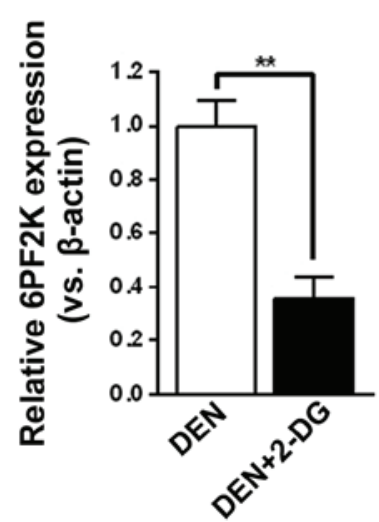

F

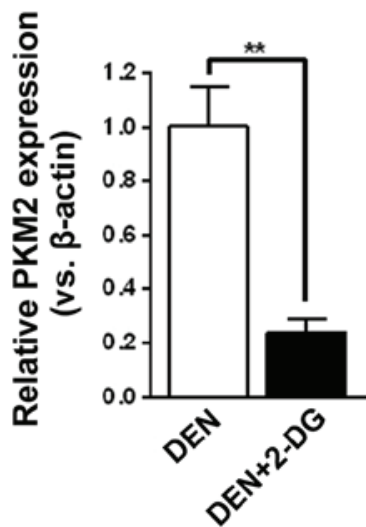

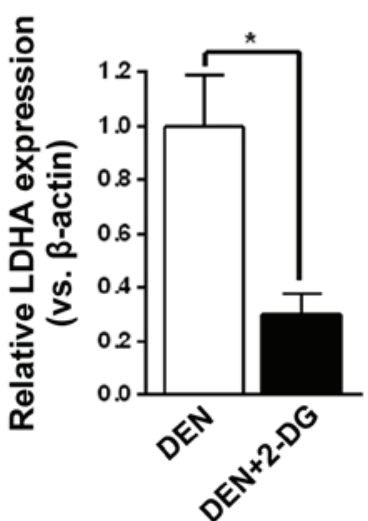

Figure 3. 2-DG inhibits tumor cell glycolysis in DEN-treated rat livers. (A-C) Levels of (A) G-6-P, (B) pyruvate and (C) lactic acid in the pericarcinous and HCC tissues of DEN and DEN+2-DG groups. Data are presented as the mean \pm standard error of the mean $\left(\mathrm{n}=3\right.$; ${ }^{*} \mathrm{P}<0.05$ and ${ }^{* * *} \mathrm{P}<0.01 \mathrm{vs}$. DEN group). (D-G) Relative mRNA expression (versus $\beta$-actin) of (D) HK2, (E) 6PF2K, (F) PKM2 and (G) LDHA in the HCC tissues of DEN and DEN+2-DG groups. Data are presented as the mean \pm standard error of the mean $\left(\mathrm{n}=3\right.$; ${ }^{*} \mathrm{P}<0.05$ and ${ }^{* *} \mathrm{P}<0.01 \mathrm{vs}$. DEN group). DEN, N-diethylnitrosamine; 2-DG, 2-deoxy-D-glucose; HCC, hepatocellular carcinoma; HK2, hexokinase 2; 6PF2K, 6-phosphofructo-2-kinase; PKM2, pyruvate kinase M2; LDHA, lactate dehydrogenase A; G-6-P, glucose-6-phosphate.

cell proliferation and higher levels of activated caspase-3 and cleaved poly ADP-ribose polymerase 1 (PARP1), another cell apoptosis indicator, than those of the DEN group (Fig. 2C and D). These data demonstrated that 2-DG led to a decrease in cell proliferation and an increase in cell apoptosis in the DEN-induced HCC tissues in the rats.

2-DG efficiently inhibits glycolysis in the HCC tissues of $D E N$-treated rats. The mechanisms by which 2-DG decreased tumor cell proliferation and survival time in hepatocarcinogenesis were further investigated. Initially, it was examined whether 2-DG effectively suppressed tumor cell glycolysis during $\mathrm{HCC}$ development in DEN-treated rats. The results demonstrated that HCC tissues of the DEN+2-DG group had lower levels of glycolysis products, G-6-P, pyruvate and lactic acid, as compared with those of the DEN group. However, 2-DG had no notable effect on the levels of glycolysis products of pericarcinous tissues in the DEN-treated rat livers (Fig. 3A-C). By contrast, treatment with 2-DG resulted in a compensatory increase in HK2 mRNA expression and decrease in mRNA expression of other glycolysis enzymes, including 6PF2K, PKM2 and LDHA, in the DEN-induced HCC tissues (Fig. 3D-G). The present results demonstrated that 2-DG prominently inhibited glycolysis in the HCC tissues of DEN-treated rats.

\section{2-DG suppresses cell metabolism and promotes autophagic} activation in HCC tissues. Subsequently, it was investigated whether glycolysis inhibition resulting from 2-DG had an impact on the other cell metabolism pathways. It was found that the levels of acetyl-CoA, citrate and isocitrate, the three intermediates of the tricarboxylic acid (TCA) cycle, malonyl-CoA, the crucial intermediate of fatty acid biosynthesis, HMG-CoA, the crucial intermediate of cholesterol biosynthesis and ATP in the HCC tissues of the DEN+2-DG group were lower than those of the DEN group (Fig. 4A-F). The present results demonstrated that 2-DG led to inhibition of the TCA cycle, fatty acid and cholesterol biosynthesis and energy generation in the DEN-induced rat HCC tissues.

Several studies have reported that autophagy, the degradation and recycling system, is activated to provide the substrates of biosynthesis and energy generation in response to nutritional deficiency and other stresses in cancer cells $(13,14,15)$. It was observed that 2 -DG led to a higher expression of Beclin-1, a crucial component of the autophagy pathway, in the HCC tissues of DEN-treated rats (Fig. 4G). 
A
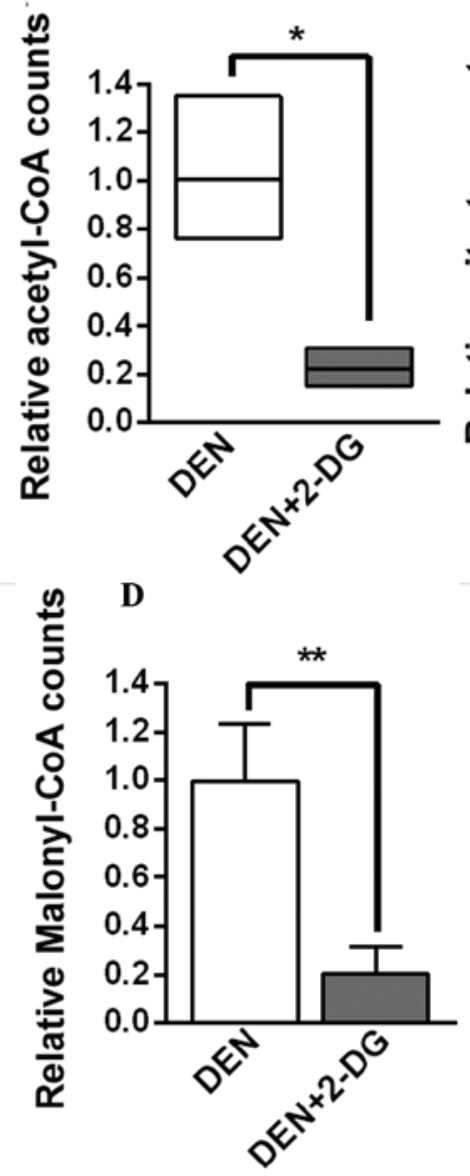

B

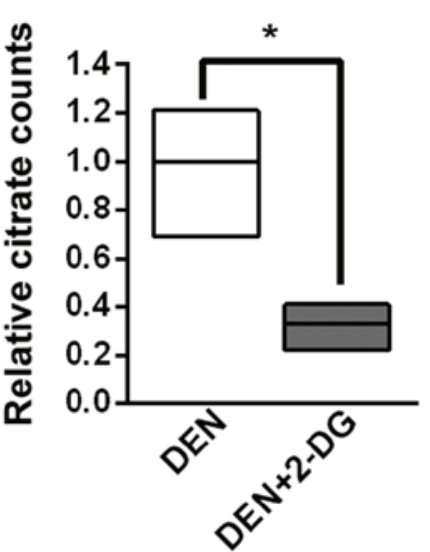

E

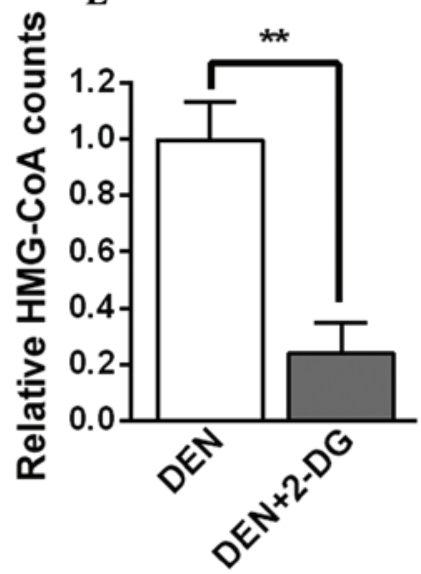

C

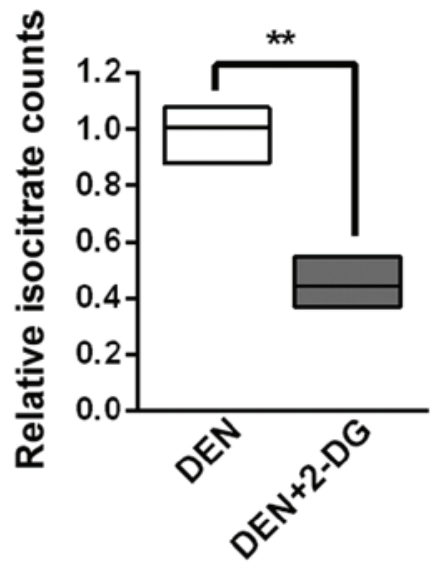

F

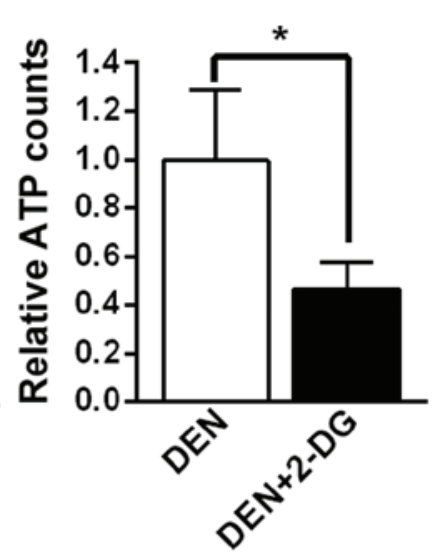

G

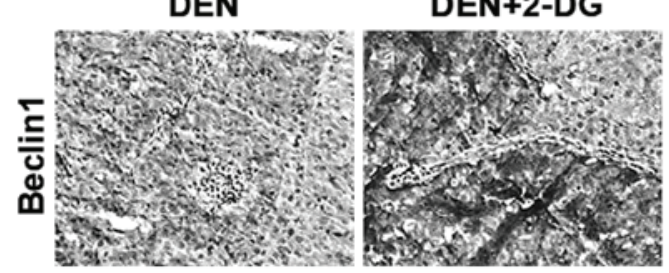

H

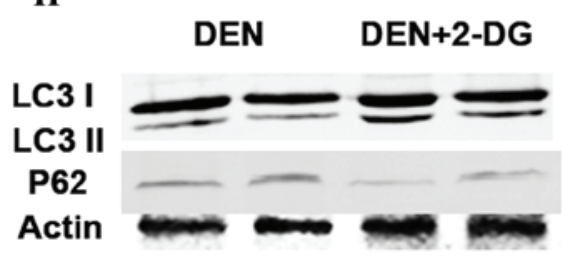

Figure 4. 2-DG inhibits TCA cycle, fatty acid and cholesterol biosynthesis and energy generation and enhances autophagy activation in the HCC tissues of DEN-treated rat livers. (A-F) Levels of (A) acetyl-CoA, (B) citrate, (C) isocitrate, (D) malonyl-CoA, (E) HMG-CoA and (F) ATP in the HCC tissues of DEN and DEN+2-DG groups. Data are presented as the mean \pm standard error of the mean $\left(\mathrm{n}=3 ;{ }^{*} \mathrm{P}<0.05\right.$ and ${ }^{* *} \mathrm{P}<0.01$ vs. DEN group). (G) Immunohistochemistry staining for Beclin-1 in the HCC tissues of DEN and DEN+2-DG groups at 17 weeks (magnification, x200). (H) Cell lysates obtained from HCC tissues of DEN and DEN+2-DG groups at 17 weeks were immunoblotted with the indicated antibodies. DEN, N-diethylnitrosamine; 2-DG, 2-deoxy-D-glucose; HCC, hepatocellular carcinoma; HMG-CoA, 3-hydroxy-3-methylglutaryl-coenzyme A; TCA, tricarboxylic acid; LC3, microtubule-associated protein 1A/1B-light chain 3; ATP, adenosine triphosphate.

Immunoblot analysis of HCC tissues suggested that the level of LC3 II was increased following treatment with 2-DG, while the level of p62 was decreased, indicating that autophagic activation was enhanced (Fig. $4 \mathrm{H}$ ). These results demonstrated that 2-DG resulted in the suppression of cell metabolism and the promotion of autophagic activation in the HCC tissues of DEN-treated rats. These severe metabolic blocks may be the reason that 2-DG led to a decrease in tumor cell proliferation and ultimately inhibited DEN-induced rat hepatocarcinogenesis.

Hypoxia enhances inhibition of cell viability, the cell cycle and tumor formation ability resulting from 2-DG in HCC cell lines. Hypoxia is an important factor in the tumor microenvironment and is present in $90 \%$ of solid tumors (16). The glycolysis pathway of cells is enhanced in the hypoxic microenvironment. The in vitro study revealed that in normal and hypoxic conditions, 2-DG led to a decrease in cell viability in the HCC cell lines (Fig. 5A and B). In addition, cell cycle analysis also revealed that 2-DG resulted in cell cycle arrest at the $\mathrm{G}_{0} / \mathrm{G}_{1}$ phase and an increase in the percentage of cells in the sub- $\mathrm{G}_{1}$ phase, an indicator of cell apoptosis, in the HCC cell lines in normal and hypoxic conditions (Fig. 5C and D). Additionally, hypoxia enhanced these effects (Fig. 5A-D). The colony formation assay also suggested that 2-DG reduced the colony number by 40 and $50 \%$ in normal 
A

SMMC7721

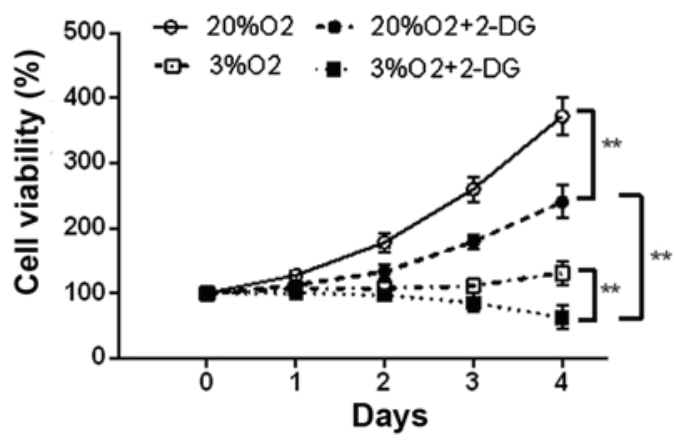

C

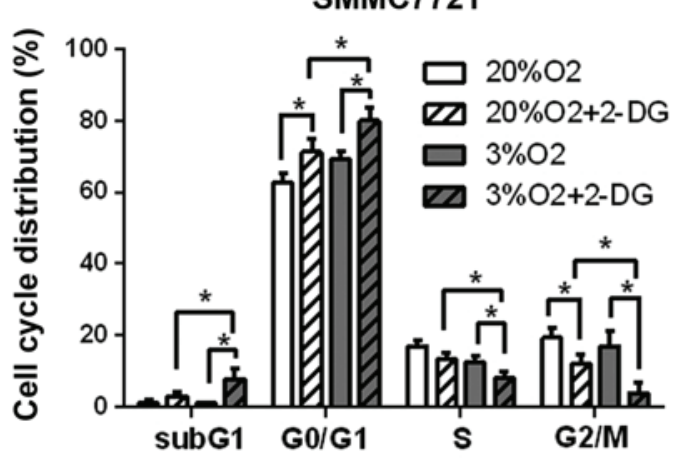

E
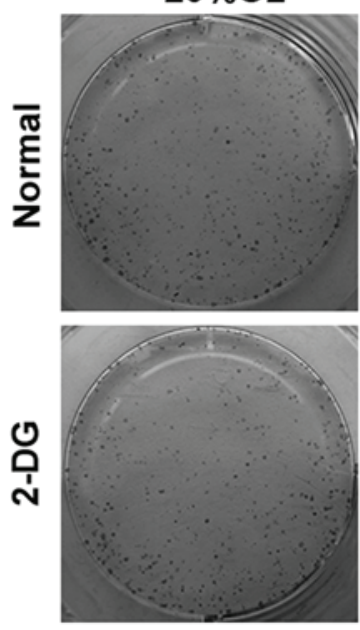

3\%O2
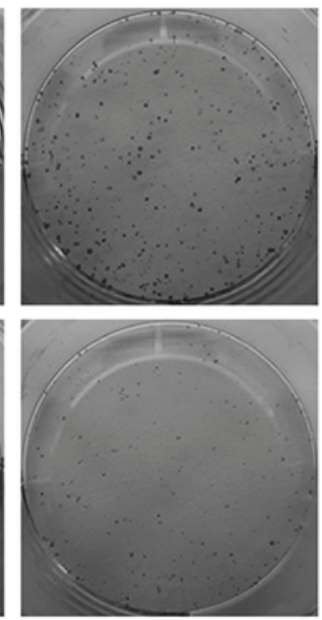

B

HepG2

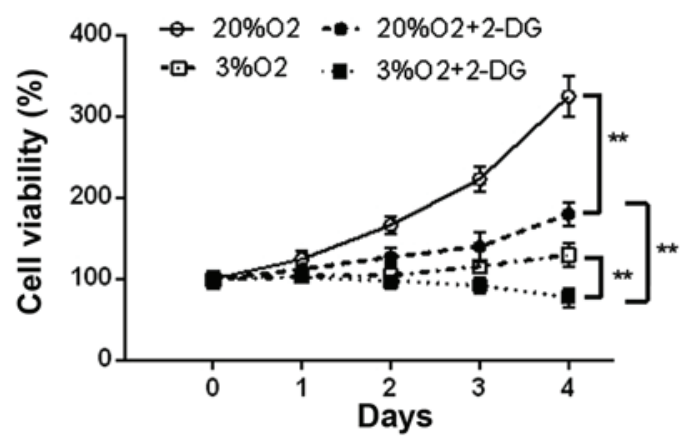

D

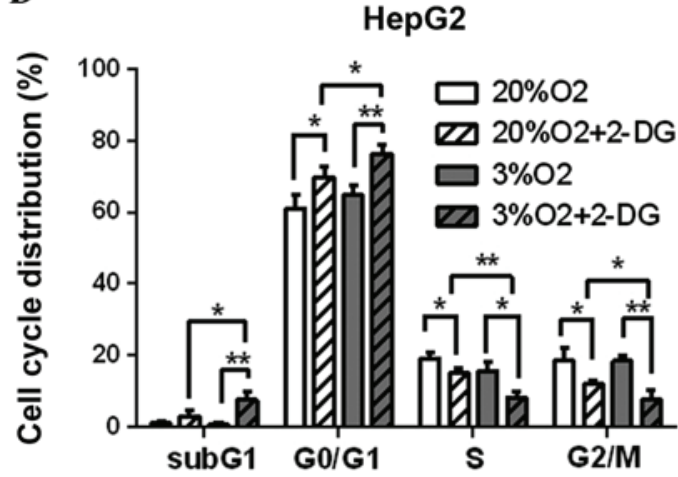

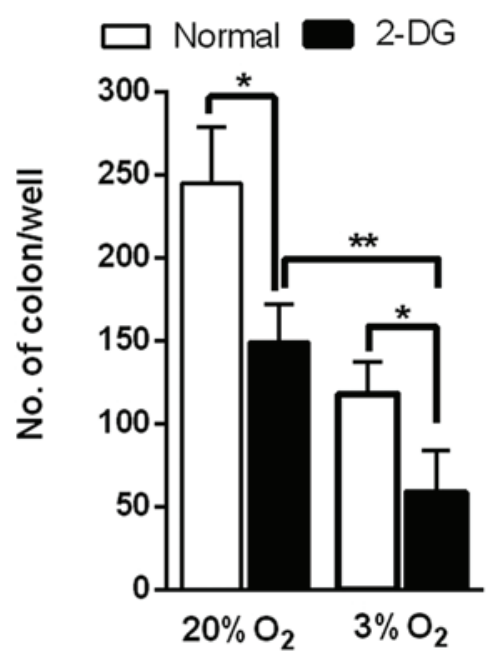

Figure 5. Hypoxia promotes 2-DG-induced inhibition of cell viability, cell cycle and colony-forming ability in hepatocellular carcinoma cell lines. (A and B) Cell viability curves of four indicated groups during 4 days evaluated by the cell counting kit- 8 assay in (A) SMMC7721 and (B) HepG2 cells. Data are presented as the mean \pm standard error of the mean $\left(n=3 ;{ }^{* *} \mathrm{P}<0.01\right)$. (C and $\left.\mathrm{D}\right)$ Cell cycle analysis of four indicated groups cells were detected by propidium iodide staining and fluorescence-activated cell sorting analysis in (C) SMMC7721 and (D) HepG2 cells. Percentages in each cell cycle phase are presented as the mean \pm standard error of the mean of three independent experiments. (E) Colony formation assay in SMMC7721 cells. Cells were seeded in 6-well culture plates (200 cells/well) and were treated by indicated methods and allowed to form colonies for 2 weeks. Colonies were stained with crystal violet and were counted and recorded. Data are presented as the mean \pm standard error of the mean $\left(n=3 ;{ }^{*} \mathrm{P}<0.05 ;{ }^{* *} \mathrm{P}<0.01\right) .2$-DG, 2-deoxy-D-glucose.

and hypoxic conditions in SMMC7721 cells, respectively. Hypoxia further led to a marked decrease $(\sim 60 \%)$ of the colony number in 2-DG-treated SMMC7721 cells (Fig. 5E). These results demonstrated that hypoxic conditions further enhance 2-DG-induced cell viability inhibition, cell cycle arrest and the reduction in tumor formation ability in the HCC cell lines.

\section{Discussion}

In the present study, it was found that 2-DG significantly delayed hepatocarcinogenesis by decreasing cell proliferation and increasing cell apoptosis in the tumor. Further investigation revealed that 2-DG not only reduced glycolysis but also suppressed the TCA cycle, fatty acid and cholesterol 
biosynthesis, ATP production and activated autophagy. In addition, a hypoxic microenvironment, an inevitable factor during tumorigenesis, may assist in improving 2-DG-induced cell viability inhibition, cell cycle retardation and a decrease in colony formation ability in hepatoma cells. These findings suggested that 2-DG may efficiently inhibit hepatocarcinogenesis in the DEN-treated rats via suppression of cancer cell metabolism.

Uncontrolled cell growth forces cancer cells to adjust their cellular metabolism. Even in the presence of oxygen, cancer cells reprogram their glucose metabolism by limiting it predominantly to glycolysis (17). This alteration is apparently counterintuitive from the angle of the efficiency of energy production, but it efficiently assists cancer cells in overcoming the obstacles of uncontrolled cell proliferation and the nutritional deficiency of the tumor microenvironment. In addition, this glycolysis-dependent metabolism was further enhanced under hypoxic conditions (5).

The current study revealed that the glycolysis inhibitor 2-DG not only affects HCC cell lines in vitro and transplanted tumor formations, but also has an inhibitory effect on primary hepatocarcinogenesis. In the DEN-induced HCC tissues, 2-DG suppressed G-6-P production by competitively occupying HK2, the first enzyme of the glycolysis pathway and subsequently led to the decrease of other glycolysis intermediates. Additionally, these alterations also led to the compensatory expression of HK2 and the reduction of other enzymes of the glycolysis pathway. Several studies have reported that enhanced glycolysis supports various biosynthetic pathways by supplying glycolysis intermediates $(18,19,20)$. It was also identified that glycolysis inhibition delayed numerous crucial biosynthetic pathways, including the TCA cycle, fatty acid and cholesterol biosynthesis and ATP production. The HCC cells may have activated autophagy in response to the inhibition of metabolism. Furthermore, 2-DG had more significant anti-tumor effects in the hypoxic environment, a crucial component in tumor development. These mechanisms are the reason that 2-DG led to a decrease of tumor cell proliferation and survival time and ultimately inhibited hepatocarcinogenesis. Notably, although 2-DG has prominent anti-tumoral effects, it had no significant effect on normal liver tissues. The reason for this may be that normal cells are not dependent on glycolysis. This finding supports a promising treatment for liver diseases, which are at risk of hepatocarcinogenesis.

In conclusion, the glycolysis inhibitor 2-DG may efficiently suppress DEN-induced hepatocarcinogenesis in the rat by reducing tumor cell proliferation and survival time via inhibition of tumor cell metabolism. This finding provides a promising approach for the prevention and treatment of HCC.

\section{References}

1. Jemal A, Bray F, Center MM, Ferlay J, Ward E and Forman D: Global cancer statistics. CA Cancer J Clin 61: 69-90, 2011.

2. Bosch FX, Ribes J, Diaz M and Cléries R: Primary liver cancer: worldwide incidence and trends. Gastroenterology 127: S5-S16, 2004.

3. Yamaguchi R and Perkins G: Challenges in targeting cancer metabolism for cancer therapy. EMBO Rep 13: 1034-1035, 2012.

4. Birsoy K, Sabatini DM and Possemato R: Untuning the tumor metabolic machine: Targeting cancer metabolism: a bedside lesson. Nat Med 18: 1022-1023, 2012.

5. Hanahan D and Weinberg RA: Hallmarks of cancer: the next generation. Cell 144: 646-674, 2011.

6. Koppenol WH, Bounds PL and Dang CV: Otto Warburg's contributions to current concepts of cancer metabolism. Nat Rev Cancer 11: 325-337, 2011.

7. Dang CV: Links between metabolism and cancer. Genes Dev 26: 877-890, 2012.

8. Takemura A, Che XF, Tabuchi T, Moriya S, Miyazawa K and Tomoda A: Enhancement of cytotoxic and pro-apoptotic effects of 2-aminophenoxazine-3-one on the rat hepatocellular carcinoma cell line dRLh-84, the human hepatocellular carcinoma cell line HepG2, and the rat normal hepatocellular cell line RLN-10 in combination with 2-deoxy-D-glucose. Oncol Rep 27: 347-355, 2012.

9. Cay O, Radnell M, Jeppsson B, Ahren B and Bengmark S: Inhibitory effect of 2-deoxy-D-glucose on liver tumor growth in rats. Cancer Res 52: 5794-5796, 1992.

10. Mack P, Ahren B, Jeppsson B, Kan Z and Bengmark S: Influence of 2-deoxy-D-glucose and arterial ischaemia on glucose oxidation and growth of liver cancer in the rat. Eur J Cancer Clin Oncol 24: 1433-1437, 1988.

11. Rajewsky MF, Dauber W and Frankenberg H: Liver carcinogenesis by diethylnitrosamine in the rat. Science 152: 83-85, 1966.

12. Sun K, Guo XL, Zhao QD, et al: Paradoxical role of autophagy in the dysplastic and tumor-forming stages of hepatocarcinoma development in rats. Cell Death Dis 4: e501, 2013.

13. Sun K, Deng W, Zhang S, et al: Paradoxical roles of autophagy in different stages of tumorigenesis: protector for normal or cancer cells. Cell Biosci 3: 35, 2013.

14. Gewirtz DA: The four faces of autophagy: implications for cancer therapy. Cancer Res 74: 647-651, 2014.

15. Lorin S, Hamai A, Mehrpour M and Codogno P: Autophagy regulation and its role in cancer. Semin Cancer Biol 23: 361-379, 2013.

16. Hockel $\mathrm{M}$ and Vaupel P: Tumor hypoxia: definitions and current clinical, biologic and molecular aspects. J Natl Cancer Inst 93: 266-276, 2001.

17. Warburg O: On respiratory impairment in cancer cells. Science 124: 269-270, 1956.

18. Zhao Y, Butler EB and Tan M: Targeting cellular metabolism to improve cancer therapeutics. Cell Death Dis 4: e532, 2013.

19. Gonzalez CD, Alvarez S, Ropolo A, Rosenzvit C, Bagnes MF and Vaccaro MI: Autophagy, Warburg, and Warburg reverse effects in human cancer. Biomed Res Int 2014: 926729, 2014.

20. Kenific CM and Debnath J: Cellular and metabolic functions for autophagy in cancer cells. Trends Cell Biol: Sept 302014 (Epub ahead of print). 